\title{
Superluminality and the equivalence postulate of quantum mechanics
}

\author{
Alon E. Faraggi ${ }^{\mathrm{a}}$ \\ Department of Mathematical Sciences, University of Liverpool, Liverpool, L69 7ZL, UK
}

Received: 17 January 2012 / Revised: 21 February 2012 / Published online: 21 March 2012

(C) The Author(s) 2012. This article is published with open access at Springerlink.com

\begin{abstract}
An interpretation of the recent results reported by the OPERA collaboration is that neutrinos propagation in vacuum exceeds the speed of light. It has been further been suggested that this interpretation can be attributed to the variation of the particle average speed arising from the Relativistic Quantum Hamilton-Jacobi Equation. I derive an expression for the quantum correction to the instantaneous relativistic velocity in the framework of the relativistic quantum Hamilton-Jacobi equation, which is derived from the equivalence postulate of quantum mechanics. While the quantum correction does indicate deviations from the classical energy-momentum relation, it does not necessarily lead to superluminal speeds. The quantum correction found herein has a non-trivial dependence on the energy and mass of the particle, as well as on distance travelled. I speculate on other possible observational consequences of the equivalence postulate approach.
\end{abstract}

Recently the OPERA collaboration reported evidence for superluminal neutrino propagation from CERN to the Gran Sasso laboratory [1]. The arrival time of the muon neutrinos with average energy of $17 \mathrm{GeV}$ is earlier by $\delta \equiv$ $\left(v^{2}-1\right)=5 \times 10^{-5}$ as compared to the speed of light in vacuum, and is reported with significance level of $6 \sigma$. The OPERA claim is compatible with earlier results obtained by the MINOS experiment at FERMILAB, which measured the neutrino speed for energies around $3 \mathrm{GeV}$ and found [2] $\delta=(5.1 \pm 2.9) \times 10^{-5}$. These results are in an apparent conflict with the arrival time of the supernova SN1987A that sets a limit of $\delta<2 \times 10^{-9}$ for electron neutrinos with energies of the order of tens of MeVs [3-5].

If the OPERA results are confirmed it will indicate a departure from one of the pivotal tenants of fundamental

a e-mail: faraggi@amtp.liv.ac.uk physics. It will necessitate reexamination of the entire formulation of quantum field theories, which take the constancy of the speed of light and Lorentz invariance, as well as causality as its basic assumptions. It is therefore an opportune moment to examine how deviations from the standard formalism may arise. The OPERA publication has indeed generated an avalanche of papers that examine the experiment, the result and its potential consequences. ${ }^{1}$

It has further been suggested that superluminal speeds are obtained from a quantum version of the relativistic quantum Hamilton-Jacobi equation [23]. The quantum versions of the non-relativistic and relativistic Hamilton-Jacobi equations have been derived from the equivalence postulate of quantum mechanics [24-30]. The equivalence postulate is related to the existence of manifest phase-space duality [2430], which is also related to the classical-quantum duality proposed in [31]. It has been shown that the equivalence postulate approach produces the phenomenological characteristics of ordinary quantum mechanics, like tunnelling and energy quantisation for bound states [24-29].

In this paper I derive an expression for the quantum correction to the instantaneous relativistic velocity in the framework of the relativistic quantum Hamilton-Jacobi equation. While the quantum correction does indicate deviations from the classical energy-momentum relation, it does not necessarily lead to superluminal speeds. The quantum correction found herein has a non-trivial dependence on the energy and mass of the particle, as well as on distance travelled.

I first examine the argument ref. [23]. The starting point is the Klein-Gordon equation for a relativistic spinless free particle

$\left(-\hbar^{2} c^{2} \Delta+m^{2} c^{4}-E^{2}\right) \psi=0$.

The Relativistic Stationary Quantum Hamilton-Jacobi equation follows by setting

$\psi=\operatorname{Re}^{\frac{1}{\hbar} S_{0}}$.

${ }^{1}$ For a partial list, see e.g. [6-22]. 
$\left(\nabla S_{0}\right)^{2}+m^{2} c^{2}-\frac{E^{2}}{c^{2}}-\hbar^{2} \frac{\Delta R}{R}=0$,

where $S_{0}$ and $R$ satisfy the continuity equation

$\nabla \cdot\left(R^{2} \nabla S_{0}\right)=0$.

In terms of the quantum potential

$Q=-\frac{\hbar^{2}}{2 m} \frac{\Delta R}{R}$

and of the conjugate momentum $p=\nabla S_{0}$, the relativistic quantum Hamilton-Jacobi equation takes the form

$E^{2}=p^{2} c^{2}+m^{2} c^{4}+2 m Q c^{2}$.

In one spatial dimension the continuity equation gives

$R=\frac{1}{\sqrt{S_{0}^{\prime}}}$,

and the quantum potential $Q$ takes the form

$Q=\frac{\hbar^{2}}{4 m}\left\{S_{0}, q\right\}$,

where $\{f, q\}=\frac{f^{\prime \prime \prime}}{f^{\prime}}-\frac{3}{2}\left(\frac{f^{\prime \prime}}{f^{\prime}}\right)^{2}$ is the Schwarzian derivative of $f$. Therefore (2) and (3) reduce to the single equation

$\left(\frac{\partial S_{0}}{\partial q}\right)^{2}+m^{2} c^{2}-\frac{E^{2}}{c^{2}}+\frac{\hbar^{2}}{2}\left\{S_{0}, q\right\}=0$.

Equation (7) is functionally similar to the nonrelativistic Quantum Hamilton-Jacobi equation. Hence, its solutions take the form

$e^{\frac{2 i}{\hbar} S_{0}\{\delta\}}=e^{i \alpha} \frac{w+i \bar{\ell}}{w-i \ell}$

where $w=\psi^{D} / \psi \in R$ and $\psi$ and $\psi^{D}$ are two real linearly independent solutions of the Klein-Gordon equation (1) in the $1+1$ dimensional case. Furthermore, we have $\delta=\{\alpha, \ell\}$, with $\alpha \in R$ and $\ell=\ell_{1}+i \ell_{2}$ integration constants. The necessary condition for the existence of a solution is that $\ell_{1} \neq 0$. This requirement is equivalent to having $S_{0} \neq c n s t$, which is a necessary condition to define the term $\left\{S_{0}, q\right\}$.

The crucial issue next is how to define the time evolution of the physical system. Floyd defines time parametrisation by using Jacobi's theorem [32-37]

$$
\begin{aligned}
t-t_{0} & =\frac{\partial S_{0}}{\partial E} \\
& =\frac{\partial S_{0}}{\partial \psi} \frac{\partial \psi}{\partial E}+\frac{\partial S_{0}}{\partial \psi^{D}} \frac{\partial \psi^{D}}{\partial E}+\frac{\partial S_{0}}{\partial \ell} \frac{\partial \ell}{\partial E}+\frac{\partial S_{0}}{\partial \bar{\ell}} \frac{\partial \bar{\ell}}{\partial E},
\end{aligned}
$$

where it is assumed that constants $\ell_{1}$ and $\ell_{2}$ may depend on $E$ as well [24-30]. Two linearly independent solutions of Eq. (1) are given by

$\psi=\sin (k q) \quad$ and $\quad \psi^{D}=\cos (k q)$,

where

$k=\frac{1}{\hbar c} \sqrt{E^{2}-m^{2} c^{4}}$.
Ref. [23] then uses Eq. (9) to define a mean speed, which is given by

$$
\begin{aligned}
\frac{q}{t}= & c \frac{\sqrt{E^{2}-m^{2} c^{4}}}{E} \\
& \times \frac{|\sin (k q)-i \ell \cos (k q)|^{2}}{\ell_{1}+\frac{\sin (2 k q)}{2 q} \frac{\partial \ell_{1}}{\partial k}-\frac{\sin ^{2}(k q)}{q}\left(\ell_{1} \frac{\partial \ell_{2}}{\partial k}-\ell_{2} \frac{\partial \ell_{1}}{\partial k}\right)} .
\end{aligned}
$$

This result differs slightly from the one derived in ref. [23]. The difference being in the last term that appears in the denominator, which is absent in ref. [23]. However, this discrepancy does not affect the conclusions. From the form of Eq. (11) we can define the quantum correction

$$
\begin{aligned}
& H_{E}\left(\ell_{1}, \ell_{2} ; q\right) \\
& =\frac{|\sin (k q)-i \ell \cos (k q)|^{2}}{\ell_{1}+\frac{\sin (2 k q)}{2 q} \frac{\partial \ell_{1}}{\partial k}-\frac{\sin ^{2}(k q)}{q}\left(\ell_{1} \frac{\partial \ell_{2}}{\partial k}-\ell_{2} \frac{\partial \ell_{1}}{\partial k}\right)}
\end{aligned}
$$

to the classical relationship

$v=c \frac{\sqrt{E^{2}-m^{2} c^{4}}}{E}$.

It is then clear that the classical limit $\hbar \rightarrow 0$ corresponds to

$H_{E}(1,0 ; q)=1$.

It is then stated in [23] that in general

$H_{E}\left(\ell_{1}, \ell_{2} ; q\right)>1$.

However, this is obviously not the case. To examine the behaviour of $H_{E}$ we can study the case $\ell_{1}$ and $\ell_{2}$ are independent of $E$. In this case the last two terms in the denominator of $H_{E}$ are zero. The expression for the mean speed, Eq. (11) reduces to

$$
\begin{aligned}
\frac{q}{t}= & c \frac{\sqrt{E^{2}-m^{2} c^{4}}}{E} \\
& \times \frac{\left(\sin ^{2}(k q)+\left(\ell_{1}^{2}+\ell_{2}^{2}\right) \cos ^{2}(k q)+\ell_{2} \sin (2 k q)\right)}{\ell_{1}} .
\end{aligned}
$$

To simplify this expression further we can examine the case $|\ell|=1$, with $\ell_{1}=\cos \alpha, \ell_{2}=\sin \alpha=$, and $\alpha=$ constant $\neq$ 0 . In this case the relativistic speed is multiplied by the factor

$v=v_{\text {rel }} \frac{(1+\sin \alpha \sin (2 k q))}{\cos \alpha}$.

Since $q$ is some arbitrary distance, we can take $q=\pi /(4 k)$ in which case the quantum correction equation (12) reduces

$$
\frac{1+\sin \alpha}{\cos \alpha}
$$

Since a priori there is no reason to restrict $\alpha$ we see that in general the quantum factor $H_{E}\left(\ell_{1}, \ell_{2} ; q\right)$ is not larger than 1 . Hence, the quantum correction does not indicate, in general, the existence of superluminal motion. 
Nevertheless, OPERA data may indeed indicate deviations from the relativistic energy-momentum relation equation (13). To study this question we can use the Jacobi theorem, Eq. (9) to define the instantaneous speed in the quantum case. For this purpose we can use Eq. (7) to rewrite Eq. (9) in the form

$$
\begin{aligned}
t-t_{0} & =\frac{\partial}{\partial E} \int_{q_{0}}^{q} d x \frac{\partial S_{0}}{\partial x} \\
& =\int_{q_{0}}^{q} d x \frac{E / c^{2}-m c^{2} \partial_{E} Q}{\left(E^{2} / c^{2}-m^{2} c^{2}-2 m c^{2} Q\right)^{\frac{1}{2}}},
\end{aligned}
$$

where $q_{0}=q\left(t_{0}\right)$. The velocity is given by

$$
\frac{d q}{d t}=\left(\frac{d t}{d q}\right)^{-1}=\frac{\partial_{q} S_{0}}{E / c^{2}-m c^{2} \partial_{E} Q} \text {. }
$$

Hence, we find that the instantaneous velocity is given by

$\dot{q}=\frac{p}{\left(E / c^{2}-m c^{2} \partial_{E} Q\right)}=\frac{p c^{2}}{E\left(1-\frac{m}{E} \frac{\partial Q}{\partial E} c^{4}\right)}$.

Making the approximation

$\frac{m}{E} \frac{\partial Q}{\partial E} c^{4} \ll 1$,

we have

$\dot{q}=\frac{p}{E} c^{2}\left(1+\frac{m}{E} \frac{\partial Q}{\partial E} c^{4}\right)$.

Hence, we see that in this case the classical relativistic relation

$\dot{q}=\frac{p}{E} c^{2}$

is modified by the quantum factor

$H_{E}\left(\ell_{1}, \ell_{2} ; q\right)=\left(1+\frac{m}{E} \frac{\partial Q}{\partial E} c^{4}\right)$,

which vanishes in the classical limit $\hbar \rightarrow 0 \Rightarrow Q \rightarrow 0$, and where $Q$ is given by Eq. (4) in the higher dimensional case and by Eq. (6) in the one dimensional case.

A priori we have no reason to assume that the quantum correction term in Eq. (22), i.e.

$\frac{m}{E} \frac{\partial Q}{\partial E} c^{4}$

$\frac{\partial Q}{\partial k}=\frac{(2 k(20+8 \sqrt{2} k q \cos (2 k q)-12 \cos (4 k q)+23 \sqrt{2} \sin (2 k q)-\sqrt{2} \sin (6 k q)))}{(2+\sqrt{2} \sin (2 k q))^{3}}$.

Setting $q=(2 \pi) /(4 k)$ gives $\partial_{k} Q \approx-2.4 k$. It is noted that similar to the case of Eq. (11) the quantum correction is not necessarily larger than 1, though larger than 1 factors are clearly possible. Examining the results obtained both in Eq. (11) and (22) we conclude the quantum correction to the energy-momentum relation does lead is positive definite. In particular we have no reason to assume that the slope of the quantum potential $Q$ with respect to the energy $E$ is positive definite. Hence, we have no reason to infer that the quantum correction factor (23) is larger than 1. By using the expression given in (6) we can study this question in the case of the relativistic stationary quantum Hamilton-Jacobi equation. Using Eqs. (8), (10) and the expression for $Q(q)$ given in Eq. (6) we obtain

$$
\begin{aligned}
\frac{4 m}{\hbar^{2}} Q & (q) \\
= & \frac{k^{2}}{4\left(\cos ^{2}(k q)+\left(\ell_{1}^{2}+\ell_{2}^{2}\right) \sin ^{2}(k q)+\ell_{2} \sin (2 k q)\right)^{2}} \\
& \times\left(3-6 \ell_{1}^{2}+3 \ell_{1}^{4}+6 \ell_{2}^{2}+6 \ell_{1}^{2} \ell_{2}^{2}+3 \ell_{2}^{4}\right. \\
& -4\left(-1+\ell_{1}^{4}+2 \ell_{1}^{2} \ell_{2}^{2}+\ell_{2}^{4}\right) \cos (2 k q) \\
& +\left(1+\ell_{1}^{4}-6 \ell_{2}^{2}+\ell_{2}^{4}+2 \ell_{1}^{2}\left(-1+\ell_{2}^{2}\right)\right) \cos (4 k q) \\
& +8 \ell_{2} \sin (2 k q)+8 \ell_{1}^{2} \ell_{2} \sin (2 k q) \\
& +8 \ell_{2}^{3} \sin (2 k q)+4 \ell_{2} \sin (4 k q) \\
& \left.-4 \ell_{1}^{2} \ell_{2} \sin (4 k q)-4 \ell_{2}^{3} \sin (4 k q)\right)
\end{aligned}
$$

Similar to the analysis in the case of Eqs. (11) we can examine the corrections to the classical relationship in special cases. We first note that the case with $\ell_{1}=1$ and $\ell_{2}=0$ we have $Q \equiv 0$. Therefore in this case the classical relation is not affected. This is in agreement with the result found in Eq. (14) [23], which showed that this choice of the $\ell_{1,2}$ parameters reproduces the classical relativistic result. We note that this is in contradiction to the requirement that $Q(q) \neq 0$ always, which is a necessary consequence of the equivalence postulate. We conclude that $\left(\ell_{1}, \ell_{2}\right)=(1,0)$ is not an allowed point in the parameter space.

We can further examine the behaviour of the quantum correction to the classical result by taking other limiting cases, i.e. setting $\ell_{1}=\cos \alpha$ and $\ell_{2}=\sin \alpha$. The result is not very illuminating and we can simplify it further by setting $\alpha=\pi / 4$. Since the partial derivative $\partial_{E} k$ is positive definite, we need only focus on the partial derivative $\partial_{k} Q$. In the special case examined here we obtain to deviations from the classical result. However, the correction does not necessarily lead to superluminal speeds. The quantum contribution has a complicated dependence on the $\ell_{1}$ and $\ell_{2}$ parameters, as well as a nontrivial dependence on the energy and distance travelled through the $k$ and $q$ variables, respectively. Furthermore, as seen in Eq. (22) 
there is a flavour dependence that arises from the particle mass.

The OPERA data, if confirmed by future experiments, may indicate a paradigm shift from the established physics foundations, perhaps discerning between the equivalence postulate approach and the conventional approaches to quantum mechanics. In this respect it is also of interest to note that energy quantisation arises in the equivalence postulate approach due to the consistency requirement that the wave-function is continuous on the extended real line [2429]. This requirement is reminiscent of quantisation in compact spaces. We may therefore speculate that the equivalence postulate approach implies that the universe has a compact topology. Investigation of this question has for example been recently discussed in [38]. Observations of large angle correlations in the cosmic microwave background radiation in contemporary experiments may therefore lend support to the equivalence postulate approach to quantum mechanics. There are two key ingredients of this approach [24-30]. The first is a quadratic identity which is a manifestation of the quantum Hamilton-Jacobi equation in its non-relativistic or relativistic forms. The second is a co-cycle condition which manifests the symmetry properties of the formalism. In one dimension it is invariant under Möbius transformations of the coordinate $q$, which in one dimension uniquely fixes the quantum potential to be given by the Schwarzian derivative, Eq. (6). In higher Euclidean dimensions the cocycle condition is invariant under $D$-dimensional Möbius transformations, whereas it is invariant with respect to the $D+1$ conformal group in the case of Minkowski space [30]. This may indicate the relevance of the conformal approach to gravity. ${ }^{2}$ The conformal approach to quantum gravity generically suffers from the existence of ghosts. Ref. [43], however, argued that in the presence of certain boundary conditions the ghosts are removed and hence enabling a consistent formulation of the theory. The equivalence postulate approach to quantum mechanics, which manifests the relevance of the conformal group in $D+1$ Minkowski space, may provide a viable framework to formulate quantum gravity. OPERA data may have thus opened the door to embark on that journey.

It should be noted that superluminal speeds are counter to the prevailing expectation in elementary physics. Indeed, special relativity and quantum field theory are being tested daily in high energy scattering experiments. Following the OPERA announcement several authors highlighted the fact that if OPERA results are correct, they will necessarily conflict with the Cherenkov pair production by the superluminal neutrinos [44] and with energy conservation in pion decay kinematics [45]. A viable question is therefore how are

\footnotetext{
${ }^{2}$ See, e.g. [39-42].
}

these questions addressed in the equivalence postulate approach to quantum mechanics. The arguments put forward in [44] on particle pair creation use the classical relativistic energy-momentum dispersion relation that are modified in the equivalence postulate approach in a highly nontrivial way. While one can contemplate, as is abundantly done in the literature, a simple correction to the classical relativistic relation in the form of a power expansion in momentum over energy, it is clear from Eqs. (11) and (21) that this is not adequate in the case of the equivalence postulate approach. The modification in this case depends non-trivially on several parameters including the distance traveled. The functional dependence form of the quantum potential is shown in Eqs. (24) and (25) and requires detailed numerical analysis to study its effects. Such a preliminary study was pursued in ref. [46]. Contrary to the arguments of ref. [45] it also clear that energy conservation is not violated due to superluminal motion in the equivalence postulate approach. The reason is that in this approach energy conservation should include the contribution of the quantum potential. Energy conservation in this picture is given by Eq. (5), which includes the quantum potential. One can also ask how and whether Lorentz symmetry is violated by the modification of the classical relativistic energy momentum dispersion relation. Underlying the equivalence postulate approach in $D+1$ dimensions there is a cocycle condition, which is invariant under $D+1$ conformal transformations with respect to the Minkowski metric [30]. Hence, the equivalence postulate formalism respect Lorentz transformations in $D+1$ dimensions. However, the quantum potential may be interpreted as an intrinsic curvature term of elementary particles [24-29], and in that respect using flat coordinates may not be adequate. Additionally, a central tenet of the equivalence postulate formalism is that the action can never be a linear function of the coordinate, i.e. in one dimension $S_{0} \neq A q+B$, where $A$ and $B$ are constants. This case corresponds to the free particle with constant speed. Hence, the equivalence postulate approach entails that the rest frame is excluded from the space of allowed frames. A particle is never at rest!

Acknowledgements I would like to heartily thank Marco Matone for our collaboration over the years and for the warm hospitality extended to me and my family on numerous visits to Schio. This work was supported in part by the STFC (PP/D000416/1).

Open Access This article is distributed under the terms of the Creative Commons Attribution License which permits any use, distribution, and reproduction in any medium, provided the original author(s) and the source are credited.

\section{References}

1. T. Adam et al. (OPERA Collaboration), arXiv:1109.4897

2. P. Adamson et al. (MINOS Collaboration), Phys. Rev. D 76, 072005 (2007) 
3. K. Hirata et al., Phys. Rev. Lett. 58, 1490 (1987)

4. R. Bionta et al., Phys. Rev. Lett. 58, 1494 (1987)

5. M.J. Longo, Phys. Rev. D 36, 3276 (1987)

6. G. Cacciapaglia, A. Deandrea, L. Panizzi, arXiv:1109.4980

7. G. Amelino-Camelia et al., arXiv: 1109.5172

8. D. Fargion, D. D’Armiento, arXiv: 1109.5368

9. G. Dvali, A. Vikman, arXiv: 1109.5685

10. G. Giudice, S. Sibiryakov, A. Strumia, arXiv:1109.5682

11. C. Pfeifer, M.N.R. Wohlfarth, arXiv:1109.6005

12. J. Mageuijo, arXiv:1109.6055

13. J. Alexandre, J. Ellis, N. Mavromatos, arXiv:1109.6296

14. A. Kehagias, arXiv: 1109.6312

15. X.J. Bi, P.F. Yin, Z.H. Yu, Q. Yuan, arXiv: 1109.6667

16. P. Wang, H. Wu, H. Yang, arXiv: 1109.6930

17. P. Wang, H. Wu, H. Yang, arXiv: 1110.0449

18. R. Cowsik, S. Nussinov, U. Sarkar, arXiv: 1110.0241

19. T. Li, D.V. Nanopoulos, arXiv: 1110.0451

20. I.Y. Arefeva, I.V. Volovich, arXiv: 1110.0456

21. E. Saridakis, arXiv: 1110.0697

22. R. Brustein, D. Semikoz, arXiv: 1110.0762

23. M. Matone, arXiv:1109.6631

24. A.E. Faraggi, M. Matone, Phys. Lett. B 450, 34 (1999)

25. A.E. Faraggi, M. Matone, Phys. Lett. B 437, 369 (1998)
26. A.E. Faraggi, M. Matone, Phys. Lett. A 249, 180 (1998)

27. A.E. Faraggi, M. Matone, Phys. Lett. B 445, 77 (1998)

28. A.E. Faraggi, M. Matone, Phys. Lett. B 445, 357 (1998)

29. A.E. Faraggi, M. Matone, Int. J. Mod. Phys. A 15, 1869 (2000)

30. G. Bertoldi, A.E. Faraggi, M. Matone, Class. Quantum Gravity 17, 3965 (2000)

31. A.E. Faraggi, M. Matone, Phys. Rev. Lett. 78, 163 (1997)

32. E.R. Floyd, Phys. Rev. D 25, 1547 (1982)

33. E.R. Floyd, Phys. Rev. D 26, 1339 (1982)

34. E.R. Floyd, Phys. Rev. D 29, 1842 (1984)

35. E.R. Floyd, Phys. Rev. D 34, 3246 (1986)

36. E.R. Floyd, Phys. Lett. A 214, 259 (1996)

37. E.R. Floyd, Int. J. Mod. Phys. A 15, 1363 (2000)

38. G. Aslanyan, A.V. Manohar, arXiv: 1104.0015

39. S.L. Adler, Rev. Mod. Phys. 54, 729 (1982)

40. P.D. Mannheim, D. Kazanas, Astrophys. J. 342, 635 (1989)

41. P.D. Mannheim, arXiv: 1101.2186

42. G. 't Hooft, arXiv:1104.4543

43. J. Maldacena, arXiv: 1105.5632

44. A. Cohen, S.L. Glashow, Phys. Rev. Lett. 107, 181803 (2011)

45. R. Cowsik, S. Nussinov, U. Sarkar, Phys. Rev. Lett. 107, 251801 (2011)

46. E.R. Floyd, arXiv:1112.4779 